\title{
Preliminary Study of Distributed Fiber Optic Sensing Technologies in Hydraulic Machinery
}

\author{
Can Yao ${ }^{1,2}, X_{\text {HUANG }}^{3}$, PingYu Zhu ${ }^{4}$, Camille Sophie Brès ${ }^{1}$, Luc Thévenaz ${ }^{2}$ \\ 1 EPFL Swiss Federal Institute of Technology, Photonic Systems Laboratory, SCI-STI-IEL, Station 11, CH-1015 Lausanne, Switzerland \\ 2 EPFL Swiss Federal Institute of Technology, Group for Fiber Optics (GFO), SCI-STI-LT, Station 11, CH-1015 Lausanne, Switzerland \\ 3 WinGD AG. Schützenstrasse 1-3, CH-8401, Winterthur, Zurich, Switzerland \\ 4 School of Mechanical and Electric Engineering, Guangzhou University, Guangzhou, P.R. China \\ E-mail address: can.yao@epfl.ch, xingxing.huang@hotmail.com
}

\begin{abstract}
This paper includes a preliminary study of bringing innovative and promising distributed optical fiber sensing technologies to strain monitoring of hydraulic machinery. (c) 2018 The Author(s) OCIS codes: (060.2370) Fiber optics sensors; (280.4788) Optical sensing and sensors; (290.0290) Scattering
\end{abstract}

\section{Introduction}

Hydro-power has been playing a vital role in providing stable electricity to the electrical grid. To gain a rapid and flexible power compensation to the other green power sources such as solar and wind energy, the smart grid needs hydro-power plants to run the hydraulic machines at off-design operation conditions including start-stops, with strong structural vibrations and high alternative dynamic stresses, which decreases the hydraulic turbine runners' lifetime (Figure 1) and increases the operation cost. A large amount of various sensors are required and installed during and after the power station infrastructure construction [1-4], to carry out strain/stress measurement, fatigue analysis and life time assessment of the prototype turbine runners.

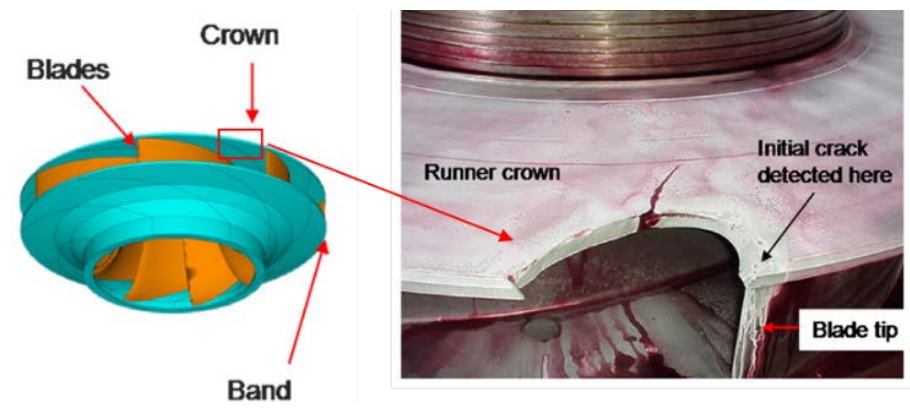

Figure 1. Sketch of a hydraulic runner and a picture of the broken runner [2].

Distributed optical fiber sensors (DOFS) are providing a more reliable, economic and efficient method for structural health monitoring, as a perfect compensation of traditional methods. To promote its application in hydraulic power plants, here we first compare different DOFS for applications in machinery fields, then present our preliminary results of the DOFS's applications in strain sensing of hydraulic turbine models.

\section{Principle and experiment}

DOFS can provide physical information continuously all along the entire optical fiber length. The DOFS for short range and high resolution strain monitoring include optical time domain reflectometry (OTDR) [5], [6] and optical frequency domain reflectometry ( $\phi$ OFDR) [7], based on Rayleigh scattering induced by the refractive index inhomogeneity in optical fiber, and the measured position distance $L$ to the detector is deduced by the travelling time of back scattered pulses $\tau$ in the optical fiber, $\tau=2 n L / c$, where $n$ is the refractive index of the fiber and $c$ is the light velocity in vacuum [8]. Table 1 shows a rough comparison of various parameters of the different DOFS.

As hydraulic turbine runners are round shaped and work under water, here we test the strain of a round disk both in air and water, by the commercial Luna ODiSI-B OFDR instrument, with a spatial resolution of $2.6 \mathrm{~mm}$. Figure 2 (a) shows the $\mathrm{S}$ shaped layout of optical fiber on the tested round disk, and the total length of the fiber is $1.1 \mathrm{~m}$ with every $5 \mathrm{~mm}$ labeled with a number from 1 to 22, so that the distributed strain measurement can be easily read out at each point along the fiber. Under a simultaneous harmonic force at the two force loading points indicated in Figure 2 (b) and (c), we get the strain distribution of the round disk along the fiber length both in air and $10 \mathrm{~cm}$ deep water (Figure 2(d) and (e)).

It can be seen that the symmetric geometry of fiber layout around the force loading points agrees very well to the symmetric strain measurement distribution along the optical fiber. The results also provide a proof of concept that the distributed optical fiber sensor can be used for under water strain monitoring. 
Table 1. A comparison of different DOFS technologies [8].

\begin{tabular}{ccccc}
\hline & Spatial resolution $(\mathrm{cm})$ & Sensing distance $(\mathrm{km})$ & Strain Sensitivity $(\mu \varepsilon)$ & Speed \\
\hline OTDR & $1-1700$ & $1-37$ & 1 & Fast \\
\$OTDR & 2.5 & $0.5-50$ & 0.001 & Slow \\
\$OFDR & 0.5 & $0.01-1$ & 1 & Fast \\
\hline
\end{tabular}
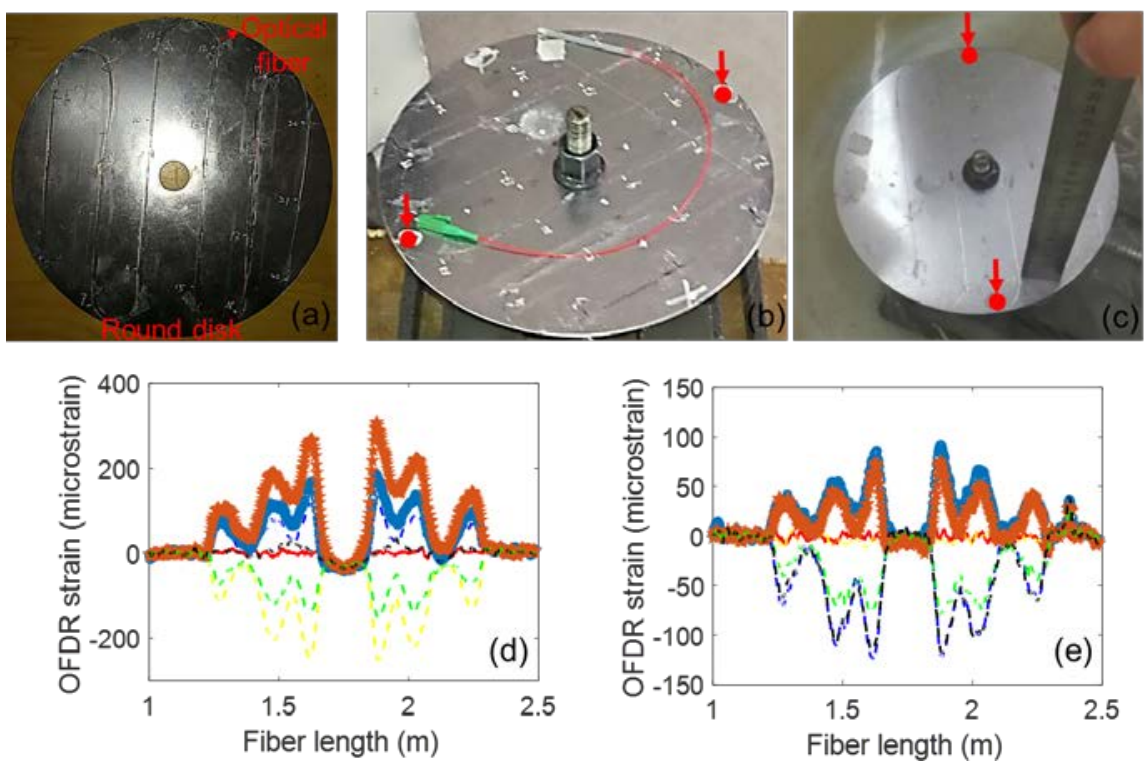

Figure 2. (a) The S shaped optical fiber layout on the tested round disk. The red force loading points indicated on the tested round disk both in (b) air and (c) water. The OFDR strain measurements along the optical fiber under the harmonic forces simultaneously loaded at the two force loading points in (d) air and (e) water.

\section{Conclusions and outlook}

In conclusion, our preliminary results conducts a proof of concept in the high potential of high precision strain measurements for hydraulic machinery by DOFS. Further investigations need to be carried out, especially challenging under the rotating state of hydraulic machinery. The fiber integration for sensing purposes also requires a dedicated expertise which is field-related, offering niche opportunities to highly specialized experts. We expect that this paper could accelerate the introduction of fiber optic sensing technology to monitor the static and dynamic behavior of hydraulic turbines in the very near future, which would deliver a huge added value to the collected information.

\section{Acknowledgments}

The authors acknowledge the Swiss National Science Foundation (SNSF) for its financial support under number PMPDP2_164532.

\section{References}

[1] X. Huang, “Contribution on the Dynamic Response of Large Hydraulic Turbomachinery Components.,” Dr. Thesis, 2011.

[2] E. Egusquiza, C. Valero, X. Huang, E. Jou, A. Guardo, and C. Rodriguez, "Failure investigation of a large pump-turbine runner," Eng. Fail. Anal., vol. 23, pp. 27-34, 2012.

[3] C. Trivedi, M. J. Cervantes, and O. G. Dahlhaug, "Experimental and numerical studies of a high-head Francis turbine: A review of the Francis-99 test case,” Energies, vol. 9, no. 2, pp. 1-24, 2016.

[4] M. S. X.Huang, C.Oram, "Stress analysis of the prototype high head Francis runner based on site measurement," 27th IAHR Symp. Hydraul. Mach. Syst., 2014.

[5] X. Lu, M. A. Soto, and L. Thévenaz, "Temperature-strain discrimination in distributed optical fiber sensing using phase- sensitive optical time-domain reflectometry,” vol. 25, no. 14, pp. 1523-1525, 2017.

[6] A. Güemes, A. Fernández-López, and B. Soller, “Optical Fiber Distributed Sensing - Physical Principles and Applications,” Struct. Heal. Monit. An Int. J., vol. 9, no. 3, pp. 233-245, 2010.

[7] J. R. Pedrazzani, S. M. Klute, D. K. Gifford, A. K. Sang, and M. E. Froggatt, "Embedded and surface mounted fiber optic sensors detect manufacturing defects and accumulated damage as a wind turbine blade is cycled to failure," Luna Innov. Inc, 2012.

[8] A. Barrias, J. Casas, and S. Villalba, "A Review of Distributed Optical Fiber Sensors for Civil Engineering Applications,” Sensors, vol. 16, no. 5, p. 748, 2016. 